\title{
COMMUNICATIONS
}

\section{LATTICE DYSTROPHY OF THE CORNEA* A CLINICAL AND MICROSCOPIC STUDY}

BY

\author{
A. J. DARK AND D. S. THOMSON \\ Nuffield Laboratory of Ophthalmology, University of Oxford
}

AT the turn of the 20th century three forms of dystrophy primarily affecting the corneal stroma were described by independent observers. All three are genetically conditioned, bilaterally symmetrical, avascular degenerations which become manifest after birth and produce progressive impairment of vision. They are thus classified as abiotrophies (Gowers, 1902). Two of these "classical" dystrophies, the lattice and granular forms, are transmitted as dominants, the third, macular dystrophy, being inherited recessively. Comprehensive reviews of the literature pertaining to these dystrophies have been compiled by Bücklers (1938), Franceschetti, Klein, Forni, and Babel (1950), and, more recently, the lattice type in particular has been reviewed by Ramsay (1957).

The separate identity of the two dominant forms had been questioned until Bücklers (1938) convincingly demonstrated their individuality. He showed that past confusion had arisen chiefly because of lack of appreciation of the changing morphology of the granular form with age; the early stages in particular closely resemble the lattice form.

Microscopically, in both conditions, a deposition of amorphous hyaline material of unknown composition (possibly similar in both forms) is found in the anterior layers of the corneal stroma. The aetiological significance of such changes is quite unknown.

A trephine corneal disc from a case of lattice dystrophy recently became available and it was considered of interest to try to determine the nature of the fundamental pathology. In the following paper tinctorial and histochemical studies of the cornea are presented with clinical studies of two families affected with lattice dystrophy. To some extent the clinical and microscopic aspects are interwoven; furthermore, apart from their intrinsic interest, the clinical data are essential for correct diagnosis; their omission by some authors has led and continues to lead to confusion. For example, Wolter and Cutler (1958) have written on the corneal histopathology in a case of granular dystrophy, but the clinical information given is strongly suggestive of the macular form. 


\section{Clinical and Genealogical Study}

\section{Materials and Methods}

Details of two unrelated families are presented: one lives in Leicestershire and is designated Family "L"; the other, an Oxfordshire family, is designated Family "O".

All available members of the two families were seen and an ocular and general medical history was obtained. This was followed by a full ocular examination and, in most of the affected members, by a general medical examination. Particular attention was paid to the investigation of the cranial nerves.

The ocular examination included refraction and trial with an afocal contact lens when indicated. Detailed study of the cornea was made in the following sequence: (i) naked eye, (ii) lens and $\times 8$ loupe, (iii) ophthalmoscope with $+20 \mathrm{D}$ lens in front of the sight hole, (iv) slit-lamp microscope, magnifications ranging from $\times 10$ to $\times 40$.

In some cases corneal nerves were stained intravitally with methylene blue by the technique recently advocated by Oppenheimer, Palmer and Weddell (1958). Corneae of all affected patients were examined in ultra-violet light of 3,650 $\AA$ wavelength. The patient was seated at the slit-lamp table and ultra-violet light from an Osram black-glass mercury lamp (10 W.MBW/U) $2 \mathrm{ft}$ away from the patient was intensified by interposing a quartz condensing lens between the lamp and the cornea. This little-used technique of ocular examination (Stein, 1957) was suggested by one of the histochemical results; intense fluorescence of the lattice opacities was observed under the ultra-violet microscope.

Corneal sensitivity was evaluated by lightly touching the cornea with monofilamentous nylon suture threads of differing calibre (Nos. 1-7). Each thread was fixed to a metal holder so that 1 inch projected, and was directed so that it touched the cornea obliquely.

Clinical descriptions are confined to affected patients. One illustrative case from each family is described in detail, and the remainder are summarized.

\section{Family " $L$ "}

The pedigree of this family, covering six generations, is shown in Fig. 1 (opposite). It contains 213 members, of whom ninety have been examined. Eleven were found to be affected and reliable evidence suggests that the disease was present in a further four, now deceased. 77 members reputed to be normal were not available for examination, and there were 42 concerning whom no useful information could be obtained.

\section{Case L.III.58, Mr. J. B., born 1894 (age 65), retired butler}

Ocular History.-Recurrent attacks of pain, redness, and lacrimation lasting several days and affecting each eye separately since the age of 42 years. Defective vision, especially in the left eye, noticed at age 45. Attended Worcester Hospital and had corneae cauterized several times. No attacks for 9 years. Still reads with aid of magnifying glass. 


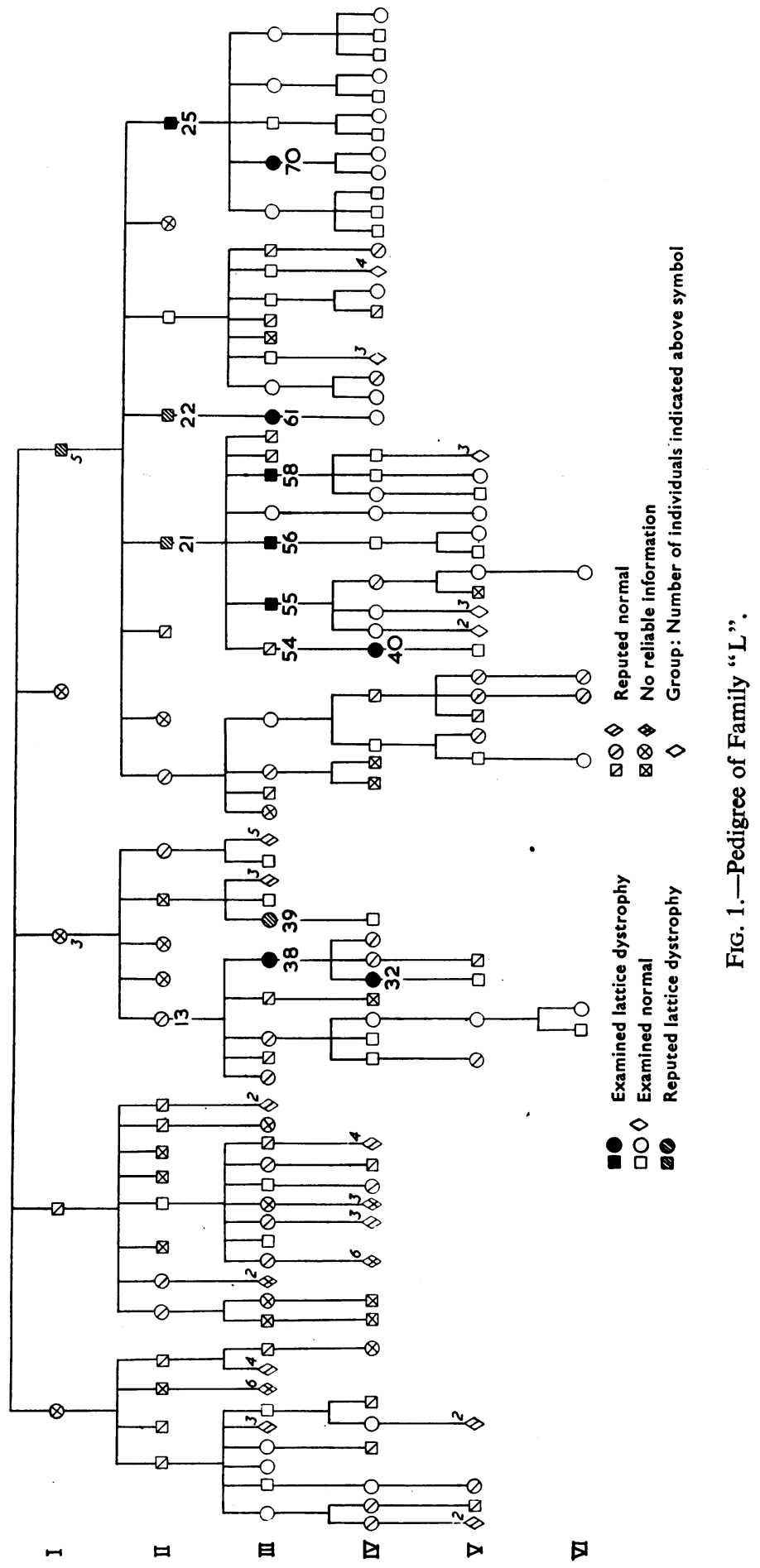


General Medical History.-Pernicious anaemia for 20 years. Increasing bilateral deafness over past 12 years, which, combined with inadequate vision, caused retirement 2 years ago.

General Physical Examination.-No abnormalities except bilateral middle-ear deafness. Cranial nerves normal.

\section{Ocular Examination}

Visual Acuity: Right Eye: 6/18 with correction; N5 with spectacles and magnifying glass.

Left Eye: Counts fingers at 2 metres. Improved to $6 / 60$ with afocal contact lens.

Conjunctivae and Sclerae: Healthy.

\section{Corneae:}

(a) Naked Eye.-Both corneae show a greyish translucent appearance with numerous striae in the zone midway between the pupil and the limbus. Considerable asymmetry exists; the dystrophy is more advanced in the left cornea, in which striae are more numerous, the anterior surface is irregularly faceted, and a faint central disc-shaped nebula is present. This correlates with the marked difference in visual acuity of the two eyes.

(b) $\times 8$ Loupe. - The lattice opacities are seen as linear striae more or less radially oriented and exhibiting occasional Y-shaped branching. Each stria shows fine calibre variations. Opacities are more numerous in the lower half of each cornea. A very faint central nebula is now also visible in the right cornea.

(c) Ophthalmoscope $+20 \mathrm{D}$ lens.-The striae are silhouetted as a brownish reticle against the red reflex. Photographs with a Zeiss-Nordensen retinal camera show this and other features described above (Figs 2 and 3).

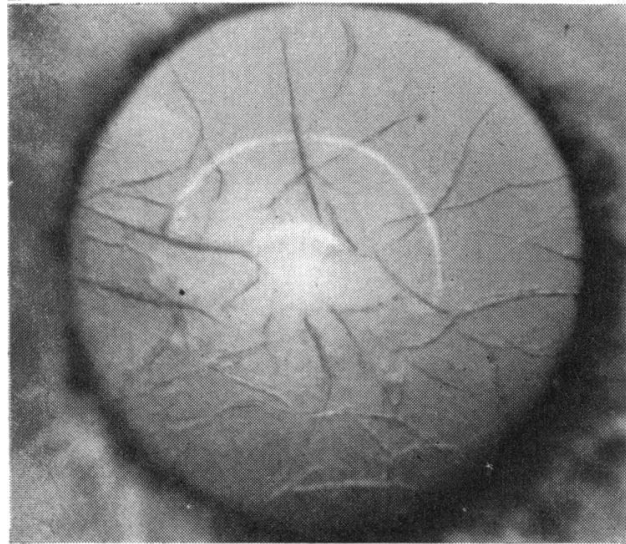

FIG. 2.-Case L.III.58, right eye (visual acuity $6 / 18$, N10 corrected), showing typical lattice striae. Anterior corneal surface smooth. The faint central disc opacity mentioned in the text is not visible.

Photograph, under full mydriasis, with Zeiss-Nordensen retinal camera. $\times 6$.

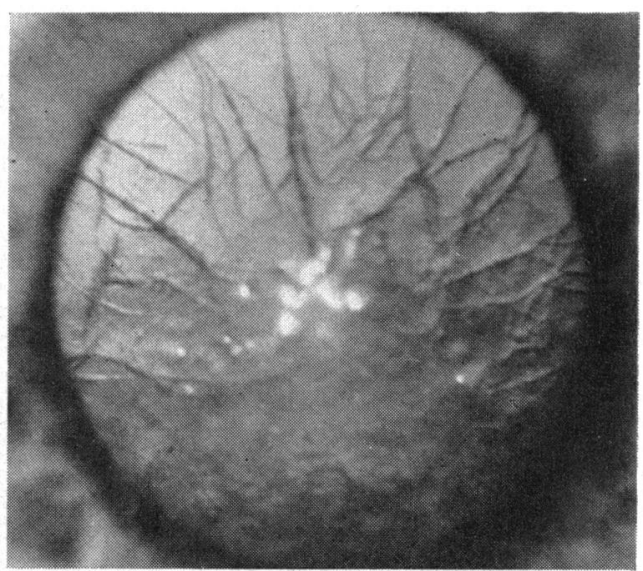

FIG. 3.-Case L.III.58, left eye (visual acuity counting fingers at $2 \mathrm{~m}$.). In comparison with the right eye (Fig. 2), the lattice opacities are thicker and more numerous. The central disc opacity is just discernible. Faceting of anterior corneal surface indicated by multiple imagery of flash.

Photograph, under full mydriasis, with Zeiss-Nordensen retinal camera. $\times 6$. 
(d) Slit Lamp.-A few punctate erosions staining with fluorescein present centrally in the right cornea; none seen in the left. In optical section the corneal epithelium rests on a relucent white seam which thickens centrally to correspond with the disc-shaped nebulae. The lattice opacities lie in the anterior half of the stroma and possess a grey homogeneous relucency. Indirect retro-illumination outlines the lattice striae, the margins towards the illuminated iris patch being dark and those away from it luminous, indicating a material of greater refractive index than substantia propria (Graves, 1924). The deeper layers of the stroma, Descemet zone and endothelium appear normal. No blood vessels are present in either cornea and limbal arcades are normal.

Corneal Sensation: Both corneae centrally insensitive to No. 7 nylon, peripherally sensitive to No. 2 nylon.

Ultra-violet Light: The lattice striae exhibit a silvery-white fluorescence; the central nebulae are not fluorescent.

Intravital Methylene Blue Staining: Corneal nerves are unstained, but the conjunctival nerves are well shown and appear normal. The lattice opacities do not stain.

\section{Case L.II.25, Mr. T. B., born 1874 (aged 85), retired railwayman}

Ocular History.-Gradual bilateral visual failure at age 40, accompanied at first by polyopia. 14 years later unable to read newsprint with magnifying glass. Recurrent attacks of redness, pain, and photophobia began at age 58. Attacks lasted 2 to 3 days and occurred two to three times a year.

General Physical Examination.-Mild parkinsonism.

\section{Ocular Examination}

Visual Acuity: Right Eye: Counts fingers at $\frac{1}{2}$ metre, not improved with spectacle lenses. Improved to $6 / 60$ with afocal contact lens.

Left Eye: Counts fingers at $1 / 3$ metre. No improvement with any type of lens.

Corneae: Both show central nebulae and lattice striae in the paracentral zone; striae are fewer and stouter than in L.III.58, they are refractile and golden yellow in colour. There are also masses of refractile yellow material aggregated beneath the epithelium, often in close relation to the lattice opacities. Similar material is distributed in a crescent along the nasal limbus of the left eye. A dense vascularized leucoma is present in the upper temporal quadrant of the left cornea.

Corneal Sensation: Absent centrally to No. 7, but present peripherally to No. 2 nylon in both corneae.

Ultra-violet Light: The lattice opacities are fluorescent but not the central nebulae or dense leucoma. Within the leucoma are embedded two lattice striae which were not visible in ordinary illumination.

Intravital Methylene Blue Staining: A few nerves in both corneae are shown; they have no connexion with lattice opacities. Several lattice striae are also coloured green or blue depending on the amount of dye absorbed. This colouration persisted for several weeks and is therefore not true intravital staining (which, characteristically, is an evanescent phenomenon), but rather simple staining of devitalized tissue.

Conjunctival nerves and Krause's end-bulbs stained and appeared normal.

Case L.III.38, Mrs. G. C. H., born 1882 (age 77)

Ocular History.-Suffered from recurrent ocular "ulceration" since age 22. Treated by Mr. Ridley at Leicester Royal Infirmary from 1906-1928. Hospital notes give diagnosis-"typical ABC keratitis" (a synonym for lattice dystrophy), and record branching striae. In 1950 perforating keratoplasty was performed on both eyes. 


\section{Ocular Examination}

Visual Acuity: Right Eye: 6/60

Left Eye: $2 / 60$, not improved with any type of lens.

Corneae: No lattice opacities now visible in either. The right graft is cloudy and surrounded by dense white scar tissue-which thins out towards the periphery. There are numerous superficial and deep vessels in the stroma. The left corneal appearance is similar, but the graft is comparatively clear. Central lens opacities are present in this eye.

Corneal Sensation: Right cornea, including graft, insensitive to No. 7 nylon; left cornea sensitive to No. 1 nylon above and No. 2 below, graft insensitive to No. 7.

Ultra-violet Light: No fluorescent material in either cornea.

Case L.III.55, Mr. J. F. B., born 1892 (age 67), miner

Ocular History.-Visual failure commenced at age 55. Mild attacks of redness from time to time.

General Health.-Excellent.

Ocular Examination

Visual Acuity: Counts fingers at $1 \mathrm{ft}$ each eye, not improved with any type of lens.

Corneae: Well marked numerous lattice striae with distinct yellow tint; they fluoresce in ultra-violet light.

Corneal Sensation: Gross impairment in both.

Case L.III.56, Mr. A. B., born 1893 (age 66), grocer

Ocular History.-Attacks of redness and visual failure from age 48. On March 22, 1958, a 4-mm. penetrating graft was done in the left eye. On April 20, 1958, a biopsy was taken of the left second costal cartilage.

General Physical Examination.-No abnormality.

Ocular Examination

Visual Acuity: Right and left, hand movements; not improved with any type of lens.

Corneae: Examination on November 20 shows findings similar to those in L.III.58. Left graft translucent and insensitive, not invaded by lattice material. Right eye is shown in Fig. 4.

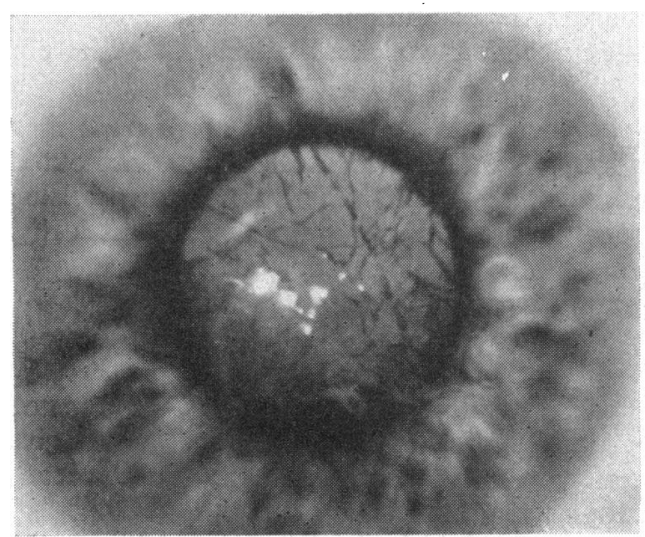

Fig. 4.-Case L.III.56, right eye (visual acuity hand movements), showing irregularity of anterior corneal surface with central disc-shaped nebula, and coarse lattice striae.

Photograph with Zeiss-Nordensen retinal camera. $\times 6$. 
Case L.III.61, Mrs. A. P., born 1900 (aged 59), housewife

Ocular History.-Noticed left eye "weaker" than right at age 35. At age 45 developed left corneal ulcer which took 5 weeks to heal. Further attacks of ulceration subsequently in both eyes. Admitted on July, 1958, to Exeter hospital with hypopyon ulcer of right cornea.

General Health.-Arthritis right knee, otherwise satisfactory.

Ocular Examination

Visual Acuity: Right Eye: 6/18, N8 with correction.

Left Eye: Counts fingers at $\frac{1}{2}$ metre; not improved with any type of lens.

Corneae: Both show characteristic striae as in L.III.58, but have distinct yellow tinge. Both show central nebulae, and the right a dense vascularized leucoma in the nasal half, the result of the corneal ulceration in July, 1958. The lattice opacities fluoresce in ultra-violet light.

Corneal Sensation: Impaired centrally to No. 7 nylon, but present peripherally to No. 4 nylon.

Case L.III.70, Mrs. A. C., born 1910 (age 49), housewife

Ocular History.-Recurrent attacks of inflammation since age 35.

General Health.-Excellent.

Ocular Examination

Visual Acuity: Right Eye: 6/60, N48 with correction.

Left Eye: 6/6, N5 with correction

Corneae: Both show features similar to those in previous cases, but changes much more advanced in right eye. Only occasional lattice opacities and no central disc in left eye.

Corneal Sensation: Impaired centrally to No. 5 nylon in both eyes. Lattice opacities faintly fluorescent.

Case L.IV.32, Mrs. L. P., born 1910 (aged 49), housewife

Ocular History.-Attacks of ocular discomfort in each eye began at age 48 . Visual failure began at the same time.

General Health.-Excellent.

Ocular Examination

Visual Acuity: Right Eye: 6/24 with correction.

Left Eye: $6 / 18$ with correction.

Corneae: Both show moderately advanced lattice dystrophy with central disc opacities, some lattice striae reach limbus.

Corneal Sensation: Retained to No. 1 nylon in both corneae.

Case L.IV.33, Mrs. H. W., born 1914, (aged 45), housewife

Chronic "conjunctivitis" since age 43.

Visual Acuity: Right Eye: 6/6.

Left Eye: $6 / 12$ with correction.

Corneae: Right shows single lattice opacity in central area. Left shows irregular net of striae covering most of the cornea. 
Case L.IV.34, Mrs. E. B., born 1916 (aged 43), housewife

Ocular Examination.-No inflammation.

Visual Acuity: Right Eye: 6/12.

Left Eye: $6 / 6$ with correction.

Corneae: Right shows several large grey lattice striae and a few finer "spider-like" opacities lying more superficially. All changes confined to temporal half of cornea. Left shows a single stout lattice opacity.

Corneal Sensation: Normal.

Ultra-violet Light: Not examined.

Case L. IV.40, Mrs. M. L., born 1921 (aged 38), housewife

No general or ocular complaint.

Visual Acuity: 6/5, N5, each eye unaided.

Corneae: Right completely normal; left shows several delicate branched lattice striae and more superficially two fine "spider-like" opacities.

Corneal Sensation: Present to No. 1 nylon.

Ultra-violet Light: Faint fluorescence of lattice striae.

The Following Members of FAMILy “L” Were Probably AfFected.

Case L.I.5, Mr. I. B., died 1909, aged 70

His son states that his father suffered from recurrent attacks of ocular inflammation from age 45 onward. Visual deterioration set in at the same time and finally became so severe that he had to give up his employment (publican).

Case L.II.21, Mr. A. B., died 1950, aged 86

Attacks of inflammation and deterioration of vision began at age 45 . When seen by an oculist in his 70's there were bilateral corneal scarring and "atheromatous ulcers". Photograph in hospital notes shows opacification of both corneae.

Case L.II.22, Mr. W. B., died 1938, aged 70

His daughter (Case L.III.61) remembers that her father suffered from recurrent ocular irritation, and says that he observed an effect like looking through a crystal in bright sunlight. He was unable to read for some years before his death.

Case L.III.39, Mr. C. B., died 1956, aged 62

Form B.D.8. (Blind Registration) records "Fair general health. Keratitis and scarring both corneae".

$$
\text { Family "O" }
$$

The pedigree of this family, covering four generations, is illustrated in Fig. 5 (opposite). There are 36 members, of whom 31 have been examined. Of these, five in Generation III were affected with typical lattice dystrophy, 25 members were found to be normal, and a further four reputed to be normal. Two cases (O.II.2 and O.I.2) were thought on evidence presented below to suffer not from lattice dystrophy, but from recurrent erosions of the cornea. 


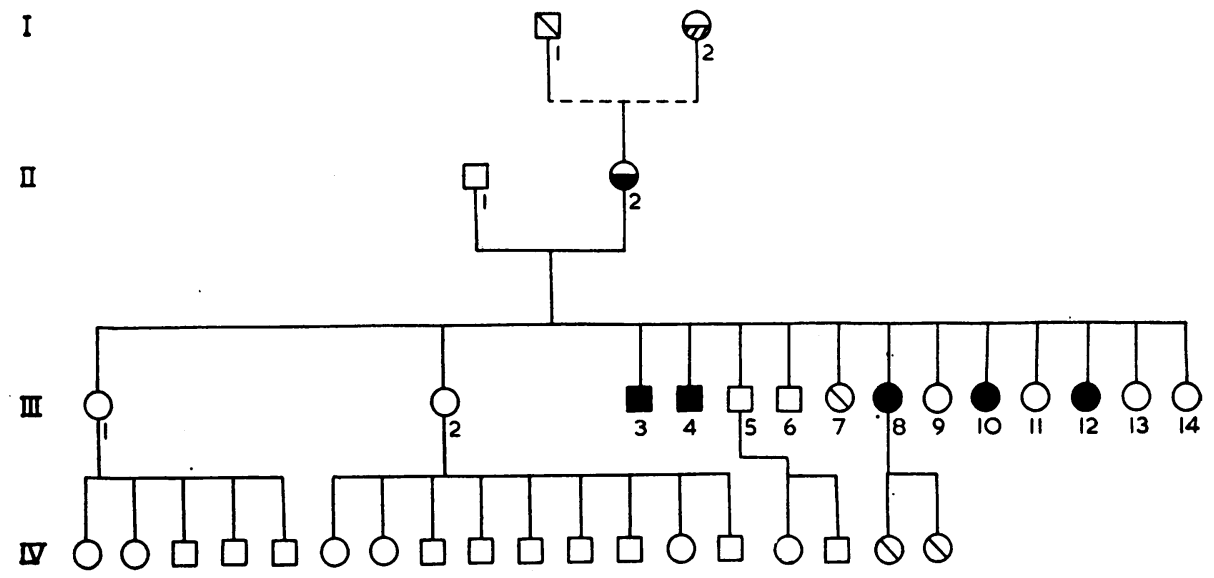

FIG. 6.-Case O.III.3, right eye (visual acuity $6 / 5$, N5 unaided), showing reticle of delicate lattice striae silhouetted in fundus glow. Anterior corneal surface smooth. Punctate opacities not visible.

Photograph, under full mydriasis, with Zeiss-Nordensen retinal camera. $\times 6$.

(2) Not examined thought to sutter from recurrent erosions

$\Delta \otimes$ Reputed normal

FIG. 5.-Pedigree of Family " $O$ ”.

\section{Case O.III.3, Mr. F. J. M., born 1927 (age 32), fitter}

Ocular History.-Recurrent acute attacks of ocular inflammation with photophobia and lacrimation affecting each eye separately began in childhood. Attacks begin on waking, last 2 to 3 days, and occur two to three times a year.

General Health.-Excellent.

Visual Acuity: Right and left eyes 6/5, N5 with correction.

Conjunctivae and Sclerae: Healthy.

Corneae:

(a) Naked Eye.-Faint central disc-like opacity and numerous very fine striae present.

(b) $\times 8$ Loupe.-Interposed between the delicate lattice striae are numerous punctate opacities-a feature not present in family " $L$ ".

(c) Ophthalmoscope.-Striae seen as fine dark threads against pupil glow (Fig. 6).

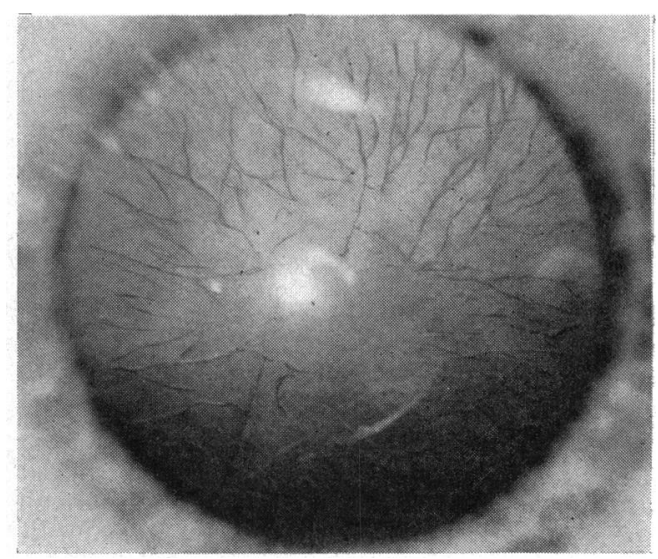


(d) Slit Lamp.-Fine lattice striae and punctate opacities have a colourless crystalline appearance in diffuse light. In optical section they are homogeneous and relucent; they lie in the anterior half of the substantia propria. A subepithelial opaque seam corresponds to the central nebula. Indirect retro-illumination reveals a similar appearance to that observed in L.III.58.

Corneal Sensation: Both sensitive to No. 4 nylon centrally and No. 2 peripherally.

Ultra-violet Light: The lattice fibres and punctate opacities show silvery-white fluorescence. The central disc is not fluorescent.

\section{Case O.II.4, Mr. E. M., born 1929 (aged 30), motor mechanic}

Ocular History.-Recurrent attacks of inflammation began at age 8 .

General Health.-Excellent.

Visual Acuity: Right Eye: 6/9, N5.

Left Eye: $6 / 12$, N8, corrected.

Corneae: Both corneae show lattice and punctate opacities which are fluorescent with central non-fluorescent nebula, as in Case O.III.3.

Corneal Sensation: Impaired, especially centrally.

Case O.III.8, Mrs. K. F., born 1936 (aged 23), housewife, married to an American soldier stationed in Hawaii.

Visual Acuity: Right Eye: 6/12.

Left Eye: $6 / 18$, corrected.

Examination by an ophthalmologist confirms history of recurrent attacks of inflammation since age 14, and presence of lattice dystrophy in both eyes.

Case O.III.10, Miss D. M., born 1939 (aged 20), typist

Ocular History: Recurrent attacks of inflammation (similar to Case O.III.3) since childhood.

Visual Acuity: Right Eye: 6/5.

Left Eye: $6 / 24$ with correction. Defective vision attributable to amblyopia ex anopsia.

Corneae: Both show punctate and lattice opacities, but central disc opacities are absent. The lattice and punctate lesions are fluorescent in ultra-violet light.

Corneal Sensation: Present to No. 1 nylon.

\section{Case O.III.12, Miss C. M., born 1943 (aged 16), schoolgirl}

Ocular Examination.-No symptoms.

Visual Acuity: 6/6, N5 each eye, unaided.

Corneae: Characteristic features of lattice dystrophy present in both, similar to Case O.III.10. Striae are fine and tend to have radial orientation.

Corneal Sensation: Tests not done.

Ultra-violet Light: Examination not done because the patient was extremely apprehensive.

Case O.II.2, Mrs. W. M., born 1902 (aged 57), housewife

Ocular History.-Recurrent attacks of ocular inflammation affecting both eyes, commenced in childhood. 
Ocular Examination:

Visual Acuity: 6/12, N8 each eye, corrected.

Corneae: Central translucent corneal opacities (deeply vascularized in both eyes) central below pupillary zone. Right opacity lying in anterior third of substantia propria shows numerous lines of clearing (Fuchs). Left opacity lies in the deeper layers of the stroma. No lattice or punctate opacities in either eye.

Corneal Sensation: Tests refused.

Ultra-violet Light: Examination shows no fluorescent material in either cornea.

Blood: Wassermann reaction and Kahn test negative.

Diagnosis: Corneal scarring following recurrent erosions of corneae.

\section{Case O.I.2, Mrs. J. E., born 1876, died 1953}

The patient is reported (by Case O.II.2, her daughter, who is a reliable witness) to have suffered from recurrent "corneal ulcers" since childhood; in spite of this, she was able to read newsprint until her death at 77 years of age. Notes made by a consultant oculist to the London Hospital in 1903 indicate "herpetic ulcers of both corneae"; no mention of corneal opacities is made, nor, unfortunately, of the visual acuity. It seems quite possible from this information that this patient also suffered from recurrent erosions of the corneae.

\section{Methods}

\section{Microscopic Study}

A full-thickness disc of corneal tissue ( $4 \mathrm{~mm}$. diam.) obtained from Case L.III.56 during keratoplasty was placed in calcium formol fixative immediately after removal and allowed to fix for 24 hours.

The disc was then cut into three approximately equal portions with a sharp razor. One third was embedded in celloidin, and the other two thirds were sectioned on the freezing microtome.

For general histological examination $7 \mu$ celloidin sections were stained with haematoxylin and eosin, May-Grunwald-Giemsa, the "Azan" method, van Gieson, Masson's trichrome, orcein for elastin, Gordon and Sweet's stain for reticulin, and Mallory's phosphotungstic acid-haematoxylin for fibrin. Tangential frozen sections $15 \mu$ thick were stained with the Bielschowsky silver method for nerve endings.

Various histological and histochemical methods were then employed for analysis of substances encountered in the diseased cornea. These techniques, which were performed on both frozen and celloidin sections, will be referred to alongside the results obtained in the analysis.

Finally, a biopsy specimen of costal cartilage was obtained, also from Case L.III.56. This was fixed in calcium formol. Celloidin sections were examined under phase-contrast, and after staining with haematoxylin-eosin and the May-Grunwald-Giemsa mixture.

\section{Results}

Histology of the Cornea.-The cornea is of normal thickness and entirely 
avascular. It shows the unique features (Figs $7,8,9$ ) characteristic of lattice dystrophy (Hermann, 1946; Paufique, Offret, and Bonnet, 1949; Franceschetti and others, 1950), viz. the typical accumulation of an unknown "hyaline" amorphous material in the anterior portion of the cornea.

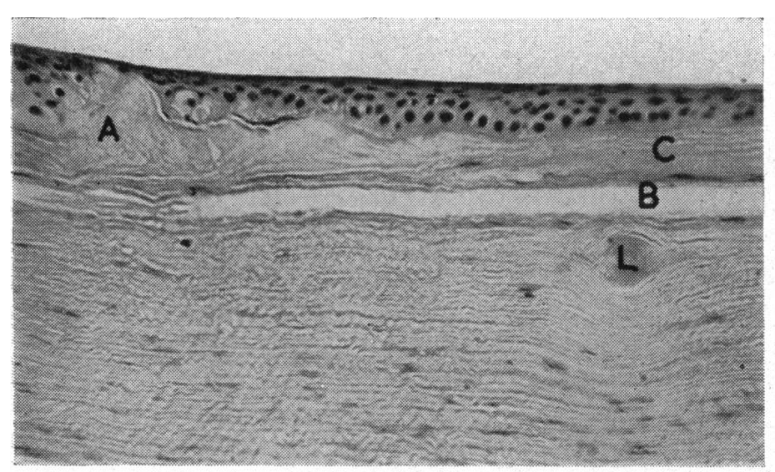

FIG. 7.-Anterior surface of epithelium is smooth. Epithelium varies greatly in thickness, conforming with irregularities in underlying stratum, which is composed of newly-formed collagen (C) (fibrillar appearance on right) and an amorphous degeneration product (A) (seen on left). Bowman's membrane (B) is almost unstained - on the left it is fibrillated and is undergoing degeneration and absorption. A lattice opacity $(L)$ is seen in crosssection, displacing adjacent corneal lamellae. Haematoxylin and eosin. $\times 510$.

FIG. 8.-Subepithelial layer (C) composed chiefly of collagen fibres, but in places containing yellow streaks of amorphous material running parallel to the surface. Bowman's membrane (B) is bordered with a yellow amorphous material. A few yellow amorphous streaks are seen in the anterior part of the substantia propria. Several lattice opacities (L) have been cut in cross-section. van Gieson. $\times 150$.
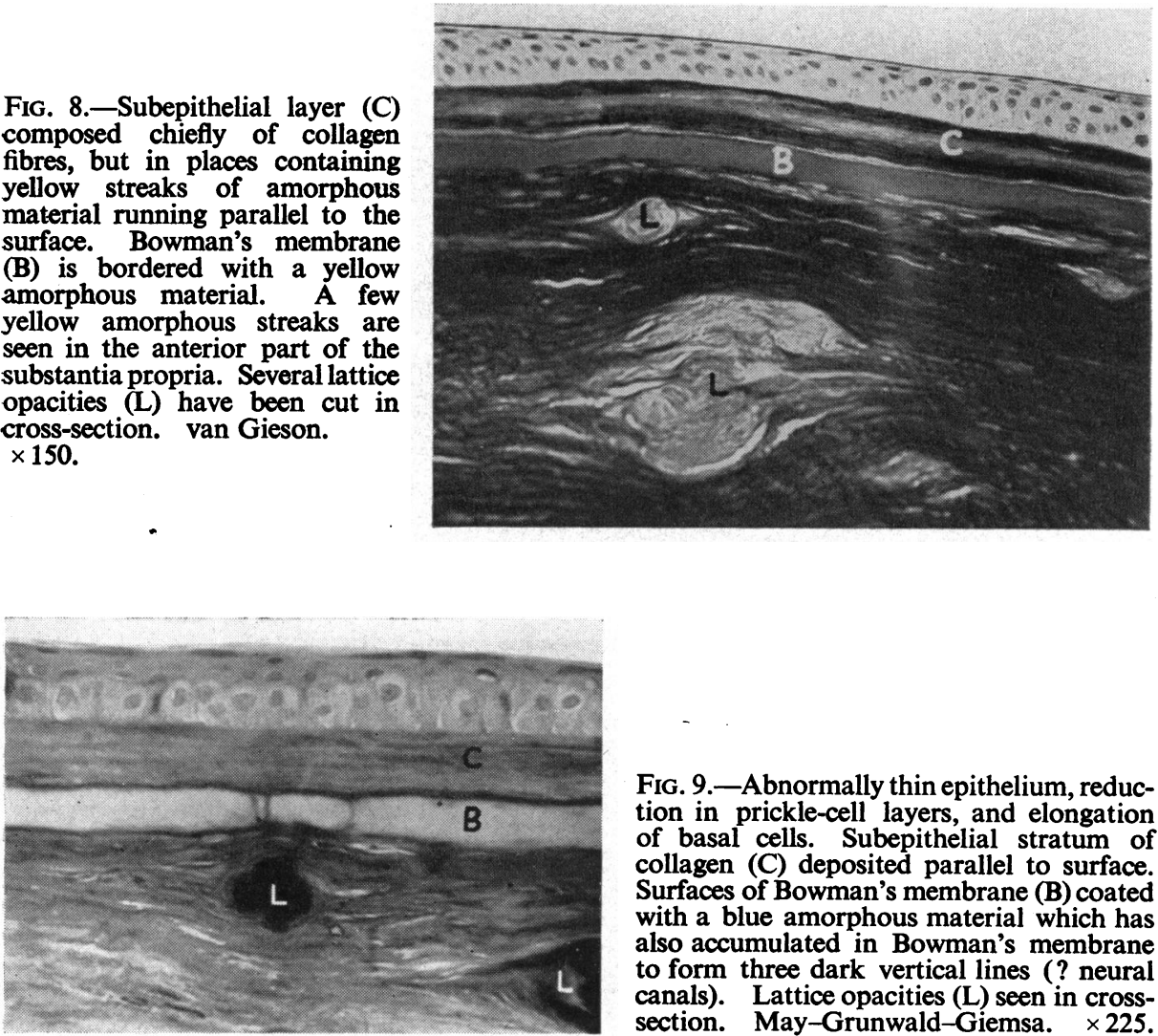

FIG. 9.-Abnormally thin epithelium, reduction in prickle-cell layers, and elongation of basal cells. Subepithelial stratum of collagen (C) deposited parallel to surface. Surfaces of Bowman's membrane (B) coated with a blue amorphous material which has also accumulated in Bowman's membrane to form three dark vertical lines (? neural canals). Lattice opacities (L) seen in crosssection. May-Grunwald-Giemsa. $\times 225$. 
Corneal Epithelium.-The anterior surface of the corneal epithelium is comparatively smooth. Variations in the height of the epithelium are conspicuous; they are due largely to variations in the number of cell layers ( 1 to 8$)$ and to a lesser degree to variations in size of the individual cells. These variations in epithelial thickness conform with irregularities in an underlying seam of amorphous material which is fully described with the substantia propria.

Epithelial squames are normal in appearance and are not keratinized. The basal cells show considerable variation in shape and arrangement; they may be considerably elongated, as in Fig. 9, or spherical with a pyknotic nucleus. The cytoplasm of this latter type of cell is practically unstained; these cells are probably degenerating. In places the epithelium is infiltrated by masses of amorphous material from the underlying layer, detached fragments of this material are found isolated within the epithelium.

Substantia Propria.-In this layer three abnormalities are conspicuous.

(1) A layer of variable thickness is interposed between the epithelium and Bowman's membrane.

(2) Bowman's membrane is undergoing dissolution and has disappeared in places.

(3) Lattice opacities are seen in transverse or oblique section as round or oval amorphous masses in the anterior half of the substantia propria (Fig. 8).

The layer between the epithelium and Bowman's membrane is largely composed of birefringent fibres running parallel to the surface. Its appearance and staining properties show it to be collagen; a few flattened fibroblast nuclei are present. An amorphous material which has different staining properties from collagen is also found in this layer. It is present for the most part as a narrow band immediately beneath the epithelium, but in places exuberant masses of this material infiltrate the epithelium, occasionally reaching almost to the surface (Fig. 7); elsewhere it forms sessile extrusions between the basal cells. Detached pieces of this material are sometimes seen lying extra-cellularly at different levels within the epithelium. These intra-epithelial extrusions and fragments doubtless reach the surface and are cast off from time to time. This amorphous material stains pink with haemotoxylin and eosin, yellow with van Gieson, red with the Masson and "Azan" methods, and exhibits blue basophilia in May-Grunwald-Giemsa preparations; it is mostly unstained with Mallory's phosphotungstic acid-haematoxylin, but occasional blue-black streaks and granules are visible. Phase-contrast microscopy confirms its amorphous nature. It is monorefringent in polarized light. Streaks and bands of this amorphous material are also seen within the collagen of this layer (Fig. 8).

Large portions of Bowman's membrane have totally disappeared. In places it is fibrillated and obviously undergoing absorption (Fig. 7). Its normal affinity for dyes is greatly reduced-so that with most stains it exhibits a marked "pallor", rendering it conspicuous by contrast to the collagen on either side (Figs 7 and 9). Both surfaces of Bowman's membrane are covered with a more or less complete but thin layer of material (Fig. 9), possessing staining properties identical with those just described for the amorphous substances associated with the sub-epithelial 
layer. This material is also found within Bowman's membrane as fine vertical streaks, possibly corresponding to the neural canals (Fig. 9) and as irregular clumps.

Conspicuous masses corresponding to the lattice opacities are found exclusively in the anterior half of the corneal stroma. They displace and distort the adjacent lamellae "like the pylons of a bridge disturb the flow of a river" (Offret, 1955). These apparently homogeneous deposits stain in a manner similar to that of the amorphous material deposited more superficially in the cornea, and described above. However, they differ in a single important respect, viz. the reticulin method demonstrates that, far from being homogeneous or "hyaline" deposits, as described by most previous investigators, there is embedded in the amorphous material a dense meshwork of fine unbranched black fibrils (Fig. 10). Some of these fibrils are aggregated into bundles which are seen to be directly continuous with the adjacent stromal fibres. A few of the larger bundles of fibrils can be seen with phase contrast. These appearances strongly suggest degeneration of collagen fibres with resultant loss of their normal staining properties and the concurrent development of an argyrophilia identical to that possessed by reticulin. Most of these fibrils are monorefringent in polarized light, indicating a physical alteration in the axial alignment of the constituent collagen molecules, but a few bundles retain their birefringence, especially near their connexion with the adjacent normal collagen. The amorphous material itself appears to be monorefringent.

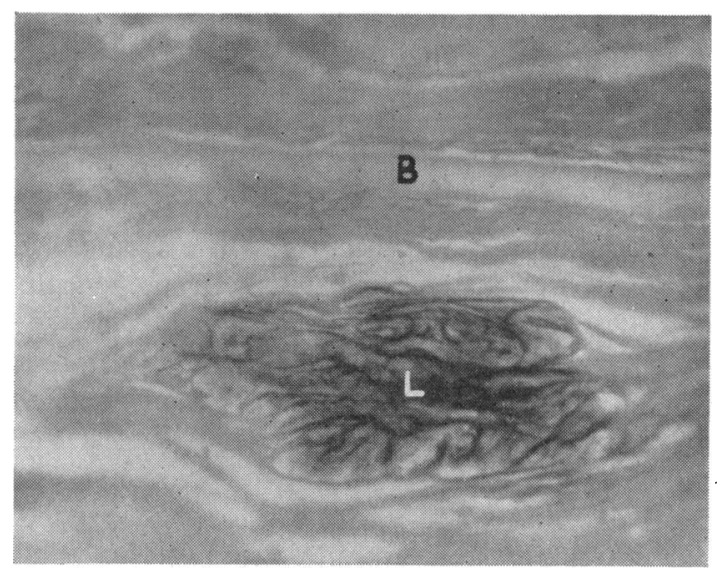

FIG. 10.-Obliquely sectioned lattice opacity (L) containing dense meshwork of argyrophil fibrils, some of which are aggregated into bundles continuous with the adjacent stromal fibres. Reticulin stain. $\times 225$.

Apart from the prominent lattice masses, small bands of amorphous material streak some of the anterior stromal lamellae, running parallel to the corneal surface (Fig. 8). They stain in the same way as the other amorphous substances already described. These streaks are more clearly visible and perhaps more numerous in preparations stained with Masson's trichrome. 
In thick unstained frozen sections $(40 \mu)$, the lattice material composing the striae has a pale yellow tint, which is not bleached after $24 \mathrm{hrs}$ in $100 \mathrm{vol}$. strength hydrogen peroxide.

In general the cell population of the anterior stroma appears somewhat reduced. A few nuclei (? macrophages) are sometimes seen aggregated on one or both sides of the lattice material, but this is not a regular feature and inflammatory cells are not visible elsewhere. The posterior half of the stroma is normal.

Descemet's Membrane and Endothelium.-These are normal.

Results with Bielschowsky Silver Method.-Most of the sections contain portions of well-impregnated nerves which appear normal. Nerve bundles show normal chiasmatic branching (Fig. 11) and the Schwann nuclei are healthy. Nerve fibres and endings are not seen in the epithelium, but the method is known to be precarious in this respect.

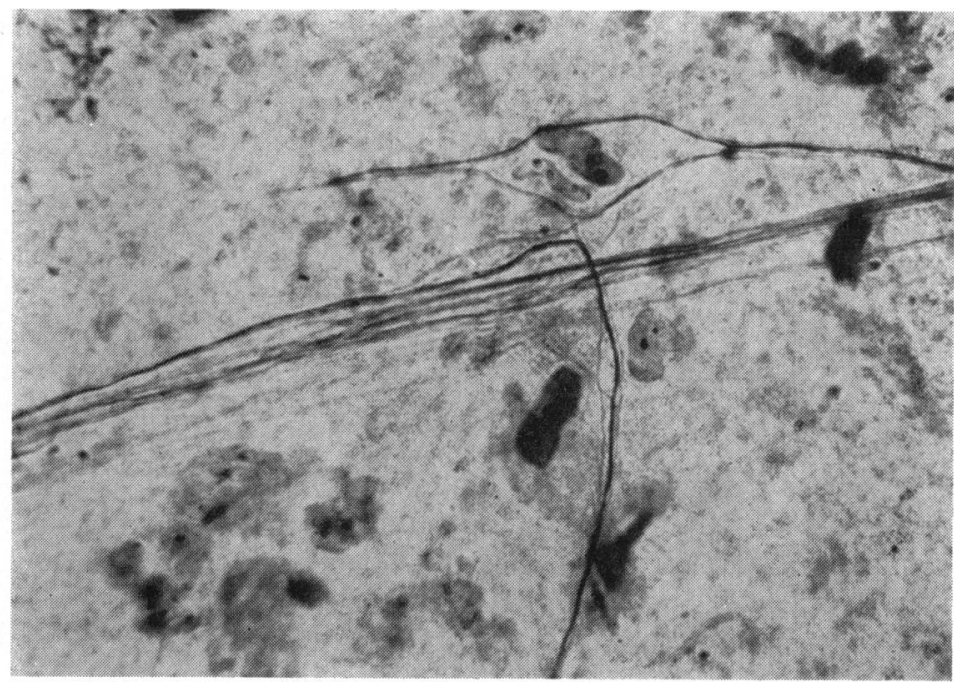

FIG. 11.-Bundle of corneal nerve fibres, showing norma lnerve fibres and chiasmatic branching of bundle. Flat section. Bielschowsky. $\times 750$.

The amorphous material composing the lattice striae is also heavily impregnated with silver, appearing dark-brown or black. However, apart from this similarity in staining, no relationship between the lattice striae and the corneal nerves can be seen.

Numerous short, linear side-processes protrude from many of the lattice striae (Fig. 12, overleaf). Immediately beneath the epithelium the lattice striae connect with a layer of black-brown deposits which have a fibrillated or stellate morphology (Fig. 12). This material corresponds with the anuclear "amorphous" substance found in this situation and described above. No observations could be made from this type of preparation on the amorphous materials distributed in other places and described in the sections on general histology. 


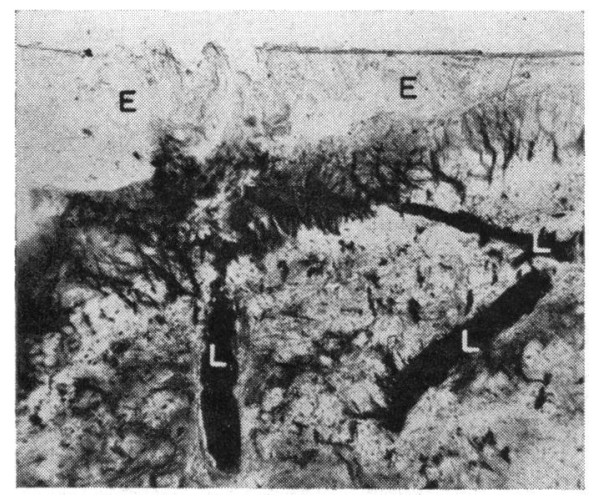

FIG. 12.-Lattice striae appear as black trunks (L) with a variable number of short rectangular side processes; two of them make contact with a layer of argyrophil material deposited in (stellate) forms which corresponds in position with the apparently amorphous material seen immediately underneath the epithelium (E). Flat section Bielschowsky. $\times 76$.

\section{Histochemical Studies}

An intimate association between accumulations of amorphous material and degenerating collagen has been noted in the general histological observations made on Bowman's membrane and the lattice opacities. Moreover, the various amorphous materials have similar staining properties and are probably related if not identical. Sections of the diseased cornea were then subjected to various microscopic techniques to determine the chemical nature of these materials, and thereby to investigate their apparent inter-relationships with each other and with the corneal stroma.

\section{(A) Cornea}

\section{FATS}

Frozen sections of the cornea were stained with Sudan Black B in 70 per cent. alcohol for periods of from $\frac{1}{2}-12 \mathrm{hrs}$ at room temperature and at $60^{\circ} \mathrm{C}$. The amorphous materials contain no sudanophilic fat.

\section{ProteINS}

(a) Amino-acid Methods.-Millon's reaction for tyrosine and Danielli's tetrazonium method for tyrosine, histidine, and tryptophan are both strongly positive, staining the amorphous materials in considerably deeper shades than that of the substantia propria. The latter method stains the amorphous materials red-brown in contrast to the stromal collagen which is red-purple.

(b) Tests for Nucleoprotein.-The Feulgen method for DNA is negative. Extraction with crystalline ribonuclease produces no loss of basophilia.

(c) Action of Proteolytic Enzymes.-Sections were incubated in crystalline trypsin and pepsin (Armour Laboratories, Ltd.) according to the methods described by Pearse (1953), and with $\alpha$-chymotrypsin* using 200 units $/ \mathrm{ml}$. for $3 \mathrm{hrs}$ at $37^{\circ} \mathrm{C}$. None of these enzymes produces any noticeable diminution in the staining reactions of the amorphous materials.

\footnotetext{
* The activity of various enzymes used was first checked on appropriate histological materials.
} 
(d) Ultra-violet Light Microscopy.-The microscopic tests performed above suggested that the amorphous protein accumulating at various sites might be a degenerate form of collagen. The fact that, certain proteins, including the "mature" collagen found for example in skin (Holborow, 1958), exhibit marked fluorescence in ultra-violet light led to the use of this method of examination. $\dagger$

In ultra-violet light of $3,650 \AA$ all the amorphous materials described above show an intense silvery-white fluorescence (Fig. 13), similar to that exhibited by " mature" collagen.

FIG. 13.-Silvery-white autofluorescence of amorphous material(s) variously deposited:

(i) immediately beneath the epithelium,

(ii) within the subepithelial seam of collagen,

(iii) edging and lying within Bowman's membrane, and forming the lattice striae (L). Descemet's membrane (D) is normally fluorescent. Ultra-violet light of $3650 \AA . \quad \times 112$.

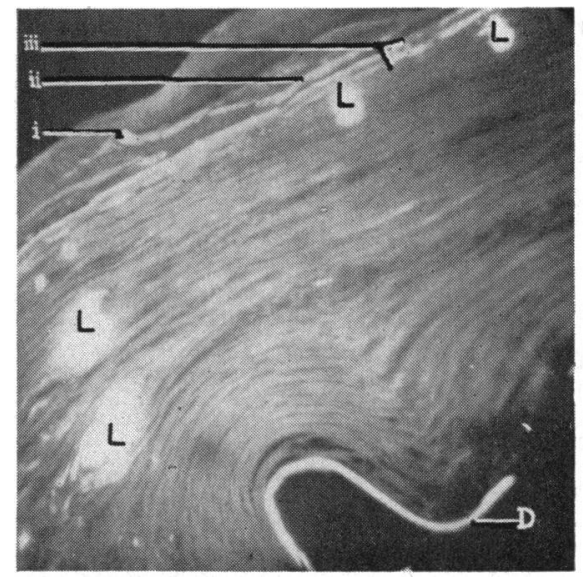

\section{CARBOHYDRATES}

An intensely positive reaction is given with PAS (the direct Schiff test is negative) indicating the presence of polysaccharides.

The results of the above tests indicate that the amorphous materials contain protein and polysaccharide mixture. This fact taken with the already established basophilic property of these materials suggested that mucopolysaccharide might be present. Tests for this were now undertaken.

\section{MUCOPOLYSACCHARIDES}

According to Dempsey, Bunting, Singer, and Wislocki (1947), the pH at which an $0.5 \mathrm{mM}$ solution of methylene blue chloride under standard conditions just fails to stain basophilic substances can be used to some extent to characterize them. The amorphous materials all fail to stain below $\mathrm{pH} 4 \cdot 5$, indicating a moderate degree of basophilia similar to that associated with corneal mucopolysaccharide.

\section{METACHROMASIA}

Aqueous toluidine blue (1/1000) stains all the amorphous materials reddishpurple, similar to that of stromal collagen. This metachromasia, like that of the stroma, is relatively alcohol-labile $(\beta)$ type and is rapidly converted to the orthochromatic form during dehydration. The remnants of Bowman's membrane

$\dagger$ Corneal collagen unlike that of sclera and 'mature' collagen elsewhere is not normally fluorescent in ultra-violet light. 
(excluding the occasional clumps and streaks of metachromatic amorphous materials which lie in it) are tinted a pale but distinctly orthochromatic blue which is in striking contrast to the underlying stroma. Normally this membrane exhibits metachromasia identical with that of the stroma-indeed it is considered to be an acellular, condensed and modified portion of the stroma. This important finding thus indicates a loss of metachromatic mucin from Bowman's membrane occurring as a preliminary to its dissolution and absorption.

\section{OTHER MUCin STAINS}

The Rinehart-Abu'l-Haj modification of Hale's method, Alcian blue 89S, mucicarmine, mucihaematin, Gram's stain and Gomori's aldehyde-fuchsin do not stain the amorphous substances or the corneal stroma.

\section{HYALURONIDASE}

Incubation with hyaluronidase (Benger "Hyalase") $1 \mathrm{mg}$. $/ 1 \mathrm{ml}$. for $3 \mathrm{hrs}$ at $37^{\circ} \mathrm{C}$ produces no reduction in metachromasia in either the amorphous materials or corneal stroma.

\section{(B) Hyaline Cartilage Biopsy}

The details of corneal histopathology just described indicate that degeneration of the stromal collagen-ground substance is probably the basic derangement in lattice dystrophy. The possibility of involvement of collagen elsewhere was considered. Since the collagen of corneal stroma and that of hyaline cartilage have important features in common, a biopsy of costal cartilage was considered justified. The specimen shows normal cartilage cells embedded in matrix which appears healthy apart from patchy deposits of calcium, a common age change in this tissue.

\section{Summary and Comment on Microscopic Observations}

The histopathology of the cornea in lattice dystrophy has been described in detail. This description differs in important respects from previous accounts.

Stansbury (1948) described vacuolation and morphological changes in the corneal epithelium; Maury (1936) found sudanophilic fat in the lattice striae; filaments in the lattice opacities were noted by Paufique and others (1949), who at first considered they were nerve fibres, but later dismissed this idea; intra-lamellar eosinophilic granules, in addition to inter-lamellar basophilic masses were noted by Hermann (1946) and Streiff (1947). Birefringence of the "foci of hyalinization of corneal lamellae" has recently been observed by Jones and Zimmermann (1959).

In the present study, epithelial changes similar to those noted by Stansbury have been confirmed; no abnormal fat was observed in the diseased cornea; the filaments noted by Paufique doubtless correspond to some of the coarser degenerating collagen strands which enter the lattice material. Frequently 
these retained their normal birefringence but the amorphous material in which they were embedded, and that found elsewhere, was monorefringent. Intralamellar eosinophilic granules were not observed.

In addition to the amorphous material which forms the bulk of the lattice striae, similar material with identical tinctorial properties is found in the epithelium and in the anterior layers of the substantia propria. Their exact distribution is described. A variety of histochemical methods confirms that all these materials are chemically similar if not identical.

Embedded in the amorphous material of the lattice striae are innumerable fine reticulin-like fibrils which are continuous with degenerating strands of corneal collagen. These were also noted by Jones and Zimmermann (1959).

The results of these histological and histochemical investigations combine to indicate that the amorphous material is composed of two substances: one, a mucopolysaccharide, has properties similar to that found normally in the corneal stroma, and the other, a protein, exhibits fluorescence in ultra-violet light resembling that of "mature" collagen. Furthermore, the distribution of these materials and their intimate association with collagen, which is obviously degenerating, indicates that the primary derangement in this condition is disease of the corneal collagen and ground substance. Two points are of special interest: that this degeneration is apparently limited to the corneal stroma, and that it apparently takes place without the direct intervention of cells.

Normal corneal nerves are seen in Bielschowsky preparations.

Biopsy of hyaline cartilage shows normal appearances.

\section{Discussion}

\section{Clinical Aspects}

An autosomal dominant mode of inheritance for lattice dystrophy is evident from a study of the pedigrees illustrated in Figs 1 and 2, a finding well established by previous observers. The clinical picture in these two families confirms that established cases show a uniform morphology, but there is a striking intra-familial constancy in the age of onset and evolution of the corneal changes. The age of manifestation was in the first decade in Family " $O$ " and in the third decade in Family " $L$ ". The fine punctate stromal opacities seen in the cases in Family " $O$ " were not seen in any case in Family " $L$ "; they may represent a true inter-familial variation.

In Family "L" (Fig. 1), fifteen members are either known or reputed to be affected with lattice dystrophy.

In two instances (L.III.38 and L.IV.40) affected members are shown as offspring of reputedly unaffected parents. This apparent anomaly is attributable in both cases to death of the parent before the age of manifestation. It is interesting that no instance of generation skipping is recorded in the classical dystrophies. 
Few of the offspring of examined affected members in Family " $L$ " appear to have inherited lattice dystrophy. It must be recorded that most of these (ten out of fourteen) are under the age of 35, the approximate age of manifestation for this particular family. It is therefore quite possible that some of them designated as unaffected will later manifest the disease.

In Family "O", Case O.II.2 shows features of special interest. No characteristic lattice opacities are present, but both cornea contain translucent central nebulae; visual acuity, although impaired, is still compatible with reading - a rare finding in patients affected with typical lattice dystrophy at this age (56 years). These facts, together with her life-long history of acute attacks of ocular inflammation, are in keeping with the diagnosis of recurrent erosions of the cornea made for this patient. The same diagnosis is suggested for her mother, Case O.I.2, although this is more tentative.

It has been noted in some families that patients affected with recurrent erosions occur alongside members who suffer from typical lattice dystrophy (Nemeth, 1935; Hermann, 1946; Schappert-Kimmijser, 1933). There is, however, no previous record of a patient so affected transmitting lattice dystrophy. It is of interest that a hereditary basis for recurrent erosions of the cornea has been established by Franceschetti (1928), who described it as an autosomal dominant in a pedigree extending over six generations.

None of the eighteen members of the fourth generation in Family " $O$ " is known to be affected. This is largely predictable, since sixteen are offspring of unaffected parents, and the other two, children of an affected mother, are probably too young to show the disease even if they carry the gene.

In Case L.IV.40 lattice opacities were unilateral and confined to a restricted area of the cornea. This patient is 37 years old and it is therefore possible that these appearances represent only an early stage in the disease. Further observation over the next few years will be required before deciding. Rare unilateral cases have been previously noted (Netchaiewa, 1937; Etienne, 1949; Ramsay, 1957) but their subsequent course has not been reported.

The characteristic corneal features of lattice dystrophy change with age. Thus, in older members of Family " $L$ ", the lattice striae are fewer in number, thicker in calibre, and in most cases have developed a distinct yellow colour (L.II.25 and L.II.55). Eventually, the lattice striae are replaced and obscured by dense white collagen, a finding illustrated by the appearance described for Case L.III.38. A disc-shaped nebula has been noted in the older members of both families. It renders the cornea translucent in the optical zone and, together with irregular faceting of the anterior corneal surfaces, causes visual impairment. Histologically the disc-shaped opacity corresponds with a layer composed of collagen and amorphous material lying between the corneal epithelium and Bowman's membrane. Irregularities in this seam are responsible for the faceting of the anterior corneal surface.

Bücklers (1938) described the lattice striae when "cut" in optical section as optically empty channels outlined by fine bright edges giving a double 
contoured appearance. Most subsequent authors have agreed with this observation, but Hermann (1946) and Ramsay (1957) have noted that some of the larger striae contain fine granular material. Subsequent authors (Franceschetti and Streiff, 1940; Hermann, 1946; Ramsay, 1957) have emphasized Bückler's observation that a "double-contoured " effect is seen in retro-illumination, and that this appearance is essential for diagnosis.

In contrast to these descriptions, in all the cases examined by us, the lattice striae in optical section appeared as solid relucent trunks; moreover, a double contour as illustrated by Franceschetti and Streiff (1940) and Ramsay (1957) was not seen by any method of illumination. Indirect retro-illumination consistently demonstrated the phenomenon of "reversed illumination", namely margins towards the illuminated iris patch were dark and those distal to it bright.

Clinical examination in ultra-violet light of $3,650 \AA$ reveals striking fluorescence of the lattice striae*. The intensity of the fluorescence appears to increase with age, a finding probably associated with increasing thickness of the striae.

\section{Aetiology}

Lattice dystrophy affects the anterior layers of the substantia propria, and it seems likely that this is the primary site of the disease. The possibility that it represents a local manifestation of generalized disease must, however, be entertained. It is reasonable to consider whether hyaline cartilage is abnormal in lattice dystrophy. Hyaline cartilage and the corneal stroma have certain common properties, viz. they are both transparent avascular structures composed of collagen fibres embedded in a matrix rich in mucopolysaccharide. However, two facts militate against a possible generalized affection of collagen, neither chondritis nor arthritis are features of the disease, and a biopsy specimen of costal cartilage taken from one of our patients showed an entirely normal structure. It is worth noting, in this connexion, that general health and longevity are not impaired in patients affected by lattice dystrophy.

On the other hand, clinical evidence supports the categorization of lattice dystrophy as an "abiotrophy", and the idea of restriction of the disease process to a specific locality will therefore be acceptable.

The problem of finding the primarily affected component of the corneal stroma would seem relatively simple. Three possibilities are the corneal nerve fibres and their Schwann cells, the corneal fibroblasts (keratoblasts), and the collagen-ground substance. These three possibilities may be considered as follows:

(1) Neurogenic Theory.-Among modern writers, Busacca (1950) and Vrabec (1957) favour a neurological origin, both incriminating the neurilemmal (Schwann) cells rather than the axons (the latter author considering that keratoblasts were also involved). Evidence in favour of this theory is three-fold:

(i) There is a striking morphological resemblance between lattice striae and corneal nerves which may be seen by comparing their form and distribution.

\footnotetext{
* Four patients in three generations with granular dystrophy were also examined by this method. In all instances the granular opacities were faintly fluorescent, so that the two dominant forms cannot be differentiated by this method.
} 
(ii) Clinically, corneal anaesthesia is a constant feature in advanced cases, as shown by the quantitative measurements made by Bücklers (1938) and Hermann (1946) and in the present work.

(iii) Vrabec (1957) illustrated his belief in the neurogenic basis of this disease with beautiful and persuasive photomicrographs, showing silvered corneal nerves apparently connecting with the lattice materials. This author also noted hyaline change in the Krause end-bulbs of the conjunctiva and impairment of the Vth, VIIth, Xth, and XIIth cranial nerves.

However, the evidence against this concept, again three-fold, is also impressive:

(i) In the present investigation only normal nerve fibres, unconnected with the lattice material, were found in silver preparations. Similar findings have been noted by Maury (1936) and Paufique and others (1949). Clinically, intravital staining of the corneal nerves with methylene blue has revealed no connexion between them and the lattice striae-neither in previous work nor in the present study - but, as Vrabec has rightly said, this method is precarious. The Krause end-bulbs and conjunctival nerves appeared normal in cases examined by us with intravital methylene blue.

(ii) Corneal hypoaesthesia may be explained other than by postulating primary disease of the nervous tissue. It may be due to extensive necrosis and fibrous tissue replacement of the superficial lamellae with secondary involvement of the nervous tissue. Most cases examined by us exhibiting corneal hypoaesthesia show a well-marked subepithelial seam of fibrous scar tissue. Corneal anaesthesia is roughly proportional with the density and thickness of this seam; thus it is most marked centrally in an area corresponding to the disc-shaped thickening of seam.

(iii) In all affected patients in the present investigation the cranial nerves were normal.

Thus it is clear from this brief consideration that the theory incriminating the corneal nerves as the primarily affected tissue component is inconclusive.

(2) Keratoblast Theory.-Lattice dystrophy may be a form of storage disease in which an amorphous material accumulates in the keratoblasts. There is little evidence for this theory and it seems likely that the changes in the number and distribution of these cells noted by some authors are secondary to the general upheaval which occurs in the anterior layers of the stroma. On the other hand, a special study of the keratoblasts, like that recently undertaken by Wolter and Cutler (1958) in "granular" dystrophy, has not been made in lattice dystrophy.

However, collagen is laid down by the keratoblasts and its metabolism is dependent upon them, so that any theory incriminating collagen must of necessity implicate them.

(3) Collagen-Ground Substance Theory.-In the present study a careful investigation of the distribution and nature (tinctorial and histochemical properties) of the abnormal material found in the diseased cornea has been made. These have been characterized as mucopolysaccharide with similar properties to those of the corneal mucin, and a protein which exhibits a fluorescence in ultra-violet light resembling that of "mature" collagen. The intimate association of this mixture of substances with degenerating collagen suggests that the primarily affected tissue is the stromal collagen and its ground substance.

Moreover, degenerating collagen fibres have been found embedded within the amorphous mixture composing the lattice striae; they stain like reticulin. This observation is reminiscent of the finding of degenerating argyrophil collagen fibres in the "fibrinoid" nodules of rheumatic fever by Glynn and Loewi (1952). However, it differs in one important respect, viz. in lattice dystrophy this degeneration has apparently occurred without direct cellular intervention.

\section{Summary}

(1) Pedigrees of two families afflicted with lattice dystrophy are presented; 121 members were examined, of whom sixteen were affected.

(2) An autosomal dominant mode of inheritance for lattice dystrophy is confirmed. 
(3) Clinical aspects are described and illustrated with photographs.

(4) Case O.II.2 in Family "O", herself affected with recurrent erosions, has transmitted typical lattice dystrophy to four of her children.

(5) In optical section, lattice striae are seen as relucent solid trunks, in contrast to the optically empty clefts described by previous authors. No "double contour" can be seen by any method of slit-lamp examination.

(6) Clinically and microscopically the lattice striae are shown to be fluorescent in ultra-violet light.

(7) Details of the histopathology in the cornea are summarized.

(8) Amorphous material in the diseased cornea has been shown histochemically to contain mucopolysaccharide and fluorescent protein.

(9) The aetiology is discussed and it is concluded that the basic pathological process is an abiotrophic degeneration of corneal collagen and ground substance.

We are greatly indebted to the following: Mrs. A. Pirie for valuable encouragement and suggestions, Mr. J. P. F. Lloyd and Mr. V. B. Purvis for permission to examine and report on their patients, Mr. A. Smith of Sunderland, Mr. W. Naunton of Norwich, Mr. A. L. McCurry of Leicester, Mr. T. D. H. Gray of London, and Col. J. N. McNair of the U.S. Army, for kindly supplying clinical records, and Mrs. M. Overall for skilful technical assistance. We are also indebted to the University of Oxford for a grant from the Ophthalmological Research Endowment Fund and to the Board of Governors of the United Oxford Hospitals for an expenses grant.

\section{REFERENCES}

BüCKlers, M. (1938). Klin. Mbl. Augenheilk., 101, Suppl. No. 3.

BusacCA, A. (1950). Quoted by Franceschetti and others (1950), p. 157.

DemPSEY, E. W., Bunting, H., SINGer, M., and WiSlOCKI, G. B. (1947). Anat. Rec., $98,417$.

ETIENNE, R. (1949). "Les hérédodystrophies familiales de la cornée." Thèse, Lyon.

FranCESCHETt, A. (1928). Z. Augenheilk., 66, 309. and StreIFF, E. B. (1940). In “Modern Trends in Ophthalmology", Series 1, ed. F. Ridley and A. Sorsby, p. 414. Butterworth, London.

KleIn, D., Forni, S., and BABel, J. (1950). "XVI Conc. Ophthal. 1950, Britannia Acta, vol. 1, p. 157.

GlynN, L. E., and LoewI, G. (1952). J. Path. Bact., 64, 329.

GOWERS, W. R. (1902). Brit. med. J., $2,89$.

Graves, B. (1924). Brit. J. Ophthal., 8, 502.

HeRmann, C. (1946). Ophthalmologica (Basel), 112, 350.

HOLBOROW, J. (1958). Personal communication.

Jones, S. T., and Zimmermann, L. E. (1959). Amer. J. Ophthal., 47, 1.

MAURY, F. H. (1936). Ibid., 19, 866.

NÉmETH, L. (1935). Klin. Mbl. Augenheilk., 95, 73.

Netchaiewa, E. (1937). Vest. Oftal., 11, 639. (Zbl. Ophthal., 1938, 41, 482).

OfFRET, G. (1955). In "Corneal Grafts", ed. B. W. Rycroft, chap. 3, p. 43. Butterworth, London.

Oppenheimer, D. R., Palmer, E., and Weddell, G. (1958). J. Anat., 92, 321.

Paufique, L., OfFret, G., and Bonnet, J. L. (1949). Bull. Soc. Ophtal. Fr., $1,14$.

Pearse, A. G. E. (1953). "Histochemistry, Theoretical and Applied". Churchill, London.

Ramsay, R. M. (1957). Trans. Amer. ophthal. Soc., 55, 701.

SCHAPPERT-KIMMIJSER, J. (1933). Klin. Mbl. Augenheilk., 90, 655.

Stansbury, F. C. (1948). Arch. Ophthal. (Chicago), 40, 189.

SteIN, L. (1957). Amer. J. Ophthal., 44, 360.

STreIFF, E. B. (1947). Schweiz. med. Wschr., 77, 339.

VRABEC, F. (1957). Ophthalmologica (Basel), 133, 160.

Wolter, J. R., and Cutler, W. M. (1958). Amer.J. Ophthal., 45, 1. 